\title{
Carta al Editor
}

\section{La automedicación como riesgo de fracaso terapéutico contra el COVID-19}

\author{
Self-medication as a risk of therapeutic failure against COVID-19 \\ Cristhian N. Rodríguez-Silva ${ }^{1,2 . a, b}$, Cynthia R. Tello-Reyna ${ }^{\text {b }}$
}

1. Escuela de Post Grado de La Universidad Nacional de Trujillo 2.Estudiante de programa Doctorado en Farmacia y Bioquímica en Universidad Nacional de Trujillo BECA CONCYTEC/FONDECYT/BANCO MUNDIAL a. Maestro en Ciencias con mención en Fisiología y Biofísica b. Químico Farmacéutico, Universidad Nacional de Trujillo

\section{Sr. Editor:}

La población mundial se encuentra a la espera de un tratamiento óptimo para la nueva enfermedad producida por el nuevo coronavirus (COVID-19) (1), puesto que aún no se tiene un tratamiento estandarizado y/o vacuna que disminuya el avance de la enfermedad, los medicamentos utilizados, solo evitan complicaciones en los pacientes hospitalizados(2). Bajo estas circunstancias, La Organización Mundial de la Salud recomendó el lavado de mano, uso de mascarillas y distanciamiento social, donde el objetivo principal sólo es evitar el incremento de la curva epidemiológica.

La falta de equipos en la Unidad de Cuidados intensivos (UCI), el hacinamiento de los cadáveres en los hospitales, el fácil acceso a los medios informativos como las redes sociales y su abundante información científica, aunque sin mucha evidencia clínica, han influenciado en generar e incrementar del pánico social nacional y distorsionar el concepto de autocuidado, manifestado en el incremento de los niveles de automedicación con el pretexto de prevenir o disminuir algunos síntomas luego de un autodiagnóstico ${ }^{(3)}$, siendo muchas veces involuntario en niños $\mathrm{y}$ ancianos(4). La automedicación está dejando de ser una práctica responsable y segura, donde el consumo inadecuado y combinaciones con otros medicamentos o alimentos, predispone al fracaso terapéutico, por lo que surge una interrogante, ¿La automedicación será genera riesgo de fracaso terapéutico contra el COVID-19?

Nuestra hipótesis es que la automedicación en las personas que consumen Azitromicina (AZT), Cloroquina, Hidroxicloroquina, Ivermectina, Paracetamol, Prednisona y Warfarina, no siempre tienen una adecuada dosificación, frecuencia de administración, reconocimiento de los eventos adversos, interacciones medicamentosas, dependencia farmacológica. Si bien los 4 primeros medicamentos pertenecen al esquema terapéutico aprobado por organismos sanitarios, aun así, su combinación y administración tiene que ser supervisada debido a los eventos adversos (5)(6), además los factores fisiológicos como la gestación y la edad y patologías preexistentes, todas estas pueden alterar las condiciones del paciente y la complicación al momento de la posible hospitalización.

Se puede predecir las consecuencias del uso inadecuado de todos estos medicamentos por ejemplo la interacción entre Azitromicina y Warfarina e hidroxicloroquina, provoca sangrado e incremento del intervalo QT; la sobredosis de paracetamol (mayor $150 \mathrm{mg} / \mathrm{Kg}$ en niños y $4 \mathrm{~g} /$ día en adultos) podría causar hepatotoxicidad y nefrotoxicidad por acumulación del metabolito $\mathrm{N}$ acetil-para-benzoquinoneimina (NAPQI), alterando la farmacocinética de otros medicamentos, además ambos, interaccionan con Warfarina, induciendo el metabolismo en el CYP 2C9 aumentando el metabolito S-Warfarina e incremento del INR respectivamente; la sobredosificación de prednisona puede generar inmunosupresión e hiperglucemia además alteración neurológica como la psicosis (7).

La manifestación de cualquiera de estos eventos complicaría en la toma de decisiones al momento de enfrentar al COVID-19. Por lo tanto, la información que se brinde al paciente y público en general debe estar destinado a la promoción de la salud, generado un impacto positivo en el autocuidado. Los profesionales de la salud principalmente Médicos y Químicos farmacéuticos deben participar de manera responsable en la orientación brindando información imparcial, asegurando el autocuidado y 
no la automedicación. Las instituciones responsables deben sancionar a toda fuente que fomente el uso irracional de medicamentos como automedicación.

\section{Agradecimiento:}

Programa doctoral financiado por FONDECYTBanco Mundial (Contrato Nº7-2018FONDECYT/BM-Programas de Doctorados en Áreas estratégicas y Generales "Doctorado en Farmacia y Bioquímica".

\section{Referencias bibliográficas:}

1. Huamán-Saavedra JJ. La pandemia del COVID-19. Rev méd Trujillo 2020;15(2):53-4

2. Kupferschmidt K, Cohen J. Race to find COVID-19 treatments accelerates. Science. (internet). 2020 (Citado el 2 de junio del 2020); 367(6485):1412-3. Disponible en: http://science.sciencemag.org/content/367/6485/1412.abstract

3. Hughes CM, McElnay JC, Fleming GF. Benefits and risks of self-medication. Drug Saf. (internet). 2001 (Citado el 2 de junio del 2020; 24(14):1027-1037. Disponible en: https://link.springer.com/article/10.2165\%2F00002018200124140-00002\#article-info
4. Valenzuela OM, Sánchez FJ, Uberos J, Checa AF, Valenzuela OC, Augustín MC, et al. Automedicación, autoprescripción y medicación «por poderes» en pediatría. An Pediatría. (internet). 2017 (Citado el 3 de junio del 2020); 86(5):264-9. Disponible

https://www.sciencedirect.com/science/article/pii/S1695403316 302065

5. Protocolo de farmacovigilancia intensiva $\mathrm{N}^{\circ}$ 03. Cloroquina, hidroxicloroquina, azitromicina como tratamiento para casos de COVID-19 moderado y severo. [Internet] Lima, Perú: EsSalud; 2020. [Citado el 20 de mayo del 2020] Disponible en: http://www.essalud.gob.pe/ietsi/pdfs/informes/20200420_Prot_ Farm_Int_COVID_19.pdf

6. Modificación del Documento Técnico: Prevención, Diagnóstico y Tratamiento de personas afectadas por COVID-19 en el Perú, aprobado por Resolución Ministerial No 193-2020MINSA, [Internet]. Diario el Peruano Normas legales 08 mayo 2020; [Citado el 20 de mayo del 2020]. Disponible en: https://img.lpderecho.pe/wp-content/uploads/2020/05/RM-2702020-Minsa-LP.pdf

7. Burger DM, te Brake LHM, Aarnoutse RE. Mechanisms of drug interactions I: Absorption, metabolism, and excretion. In: Pai MP, Kiser JJ, Gubbins PO, Rodvold KA, editors. Drug interactions in Infectious Diseases: Mechanisms and models of drug interactions. [internet]. 4th ed. Switzerland: Springer Nature; 2018. [Citado el 20 de mayo del 2020] p. 15-48. Disponible en: 